\title{
An Enhanced Approach for Quantitative Prediction of Personality in Facebook Posts
}

\author{
Azhar Imran ${ }^{\mathrm{a}, *}$, Muhammad Faiyaz ${ }^{\mathrm{b}}$, Faheem Akhtar ${ }^{\mathrm{c}}$ \\ ${ }^{a}$ School of Software Engineering, Beijing University of Technlogy, Beijing, China \\ ${ }^{b}$ Department of Computer Science \& IT, University of Sargodha, Sargodha, Pakistan \\ ${ }^{c}$ Department of Computer Science, Institute of Business Administration, Sukkar, Pakistan
}

Received: 01 November 2017; Accepted: 19 December 2017; Published: 08 March 2018

\begin{abstract}
Social media is a collection of computer-mediated technologies that encourages the creation and sharing of data, thoughts and vocation interests by means of online communities. There are various kinds of web-based social networking i.e. micro-blogs, wikis and social networking sites. Different social media like Facebook, LinkedIn, Google+ and Twitter are the popular sources for connecting people all over the globe. Facebook is one of the commonly used platform where individual's used to stay in touch, business personnel used for marketing and others used to share expedient information. Due to this lucrative nature, one's personality can be predicted on the basis of posts created, commented on others post and likes against any posts. We have developed in-house tool using python language that defines personality in terms of psychological model of Big-5 personality traits including extraversion, neuroticism, agreeableness, openness and conscientiousness. The dictionary based approach has been used in this tool in which we have combined three dictionaries (WordNet, SenticNet and Opinion Lexicon). Our proposed technique has shown promising results as we have analyzed 213 unique Facebook profiles and their results outperforms the others. Furthermore a comparative analysis of machine learning classifiers i.e. support vector machine, naïve bays and decision tree has performed. Our approach succeeds to predict personality traits. We are intended to predict personality from roman English posts in future.
\end{abstract}

Index Terms: Personality Prediction, Psychological Personality Traits, Sentiment Analysis, Social Media.

(C) 2018 Published by MECS Publisher. Selection and/or peer review under responsibility of the Research Association of Modern Education and Computer Science.

\footnotetext{
* Azhar Imran. Tel.: +86-15601260301

E-mail address: azharimran63@gmail.com
} 


\section{Introduction}

Social media is computer-mediated tool that is used for creation and sharing of data, thoughts, vocation interests and different types of articulation by means of virtual groups and systems [1]. There are many types of web-based social networking, including blogs, micro-blogs, wikis, social networking sites, podcasts, widgets and virtual worlds etc. Social networking uses web based advancements, computers and versatile innovations (e.g., cell phones and tablet PCs) to make exceedingly intelligent platforms through which people, groups and associations can share, co-make, talk about, and alter user produced content or pre-made content posted on the web. They acquaint significant and inescapable changes with correspondence among different stakeholders [2]. Different social media like Facebook, LinkedIn, Google+ and Twitter are the popular sources for connecting all over the globe. It is enormously common for both personal and professional seeks. Individual's used to stay in touch, business personnel's used for marketing and others use to share useful information.

Facebook was launched in February 4, 2004 that allows users having age above thirteen to register their account on it. According to peer analytics, Facebook has above 2 billion active users [18]. It is world's largest online community where users can join for free and stay in touch with family, share photos, chat online, find friends and share memories. Recently, SNSs such as Facebook, Twitter, LinkedIn and Google+ have become extremely famous all over the world. These online social networking sites are playing vital role to accurately define the user's personality on the bases of their social activities. Facebook has been used effectively in many real world phenomena such as promoting academic engagement [21], engaging stakeholders [22], diagnose healthcare problems [23], in entertainment [24], weather forecasting [25] and sports [26].

Facebook is public in its nature, which give freedom to its users regarding comments, posts and likes. Due to this liberty, some people undergoes in various negativities. Different users posts, comments or likes on the basis of their personal interests. This personal interest falls under the category of personality traits. The prediction of personality can be one of the most important factor of these social sites. As there is no such clear definition of personality, it is supposed to be one of the most challenging problem at the present time. One's post can be observed and analyzed as a personality traits. The Big-5 personality traits are comprehensive personality qualities including extraversion, neuroticism, agreeableness, openness and conscientiousness.

In this article, personality has predicted in Facebook status posts. This prediction is based on text mining techniques for preprocessing of data. A comparative analysis of different machine learning classifiers i.e. Support Vector Machine (SVM), Naïve Bays (NB) and Decision Tree (DT) has been performed.

In section 2, related works of personality prediction techniques has been discussed. Section 3 defines research methodologies. Experiments and results are illustrated in section 4. In section 5, conclusion and future directions of this research has been discussed.

\section{Related Works}

Social psychology investigation has highlighted that personality plays an authoritative role in the way individuals achieve the images they transfer in self-description [3]. Therefore, current researches have described that individuals can make precise personality brands from the information presented in social network individual's profiles. Researchers have examined the explicit attributes from individual profiles, text based attributes i.e. example comments and likes are extra beneficial to produce personality judgments [4]. Researcher has focused on personality prediction from slight corpora of social network data, like Twitter and Facebook, exploiting either linguistic features in status updates, social features such as friends count, and daily activity [5]. An old-fashioned method to measure personality qualities has required contributors to response a series of questions assessing their behavior and preferences, but such tactic is time intense and impracticable. Specially, virtual users might be unenthusiastic to devote their time filling-in questionnaires [6]. In a study by [7] the relationship among sentiments conveyed in Facebook status updates and the individual's age, sex and personality features were examined. It was observed that talkative and open users are extra expressive in their statuses. Researchers have found that people having positive emotions and passionately unchanging people are 
famous as well as dominant individuals on most popular micro blogging service [8]. Golbeck, J., et al., have demonstrated that an individual's Big Five identity attributes can be anticipated from general society data they share on Twitter [15]. They have used capacity value to figure a person's identity qualities, various open entryways were opened for tweaking interfaces and data. They examined some of these open gateways for publicizing and interface plot. In [16] author has developed an automated personality type prediction tool that classifies DISC identity types in light of Facebook individual profiles. This study turned to the regularly used feature grouping technique: planning the elements, screening the elements, preparing the classifier show utilizing the rest of the features, and outlining the classification method IFC appropriately with 70 to $80 \%$ precision. Author has used new data collection technique for prediction of election outcomes and a topic modeling method for extracting topics in twitter [19]. This technique is based on electoral predictions using social media data based dynamic keyword methodology. In [20] sentiment analysis and opinion mining concepts has extracted in social media. The fundamental objective behind such analyses is to determine the level of criticality with respect to criticism or appreciation described in the comments, tweets or blogs. The trend analysis techniques can also be systematically exploited for opinion making among the masses at large.

\section{Research Methodology}

To examine the personality traits, it is important to define the principles to predict the personality. Since the primary goal of our research is to predict personality in Facebook posts, there must be framework for quantitative prediction. The workflow of our proposed research has been described in fig.1.

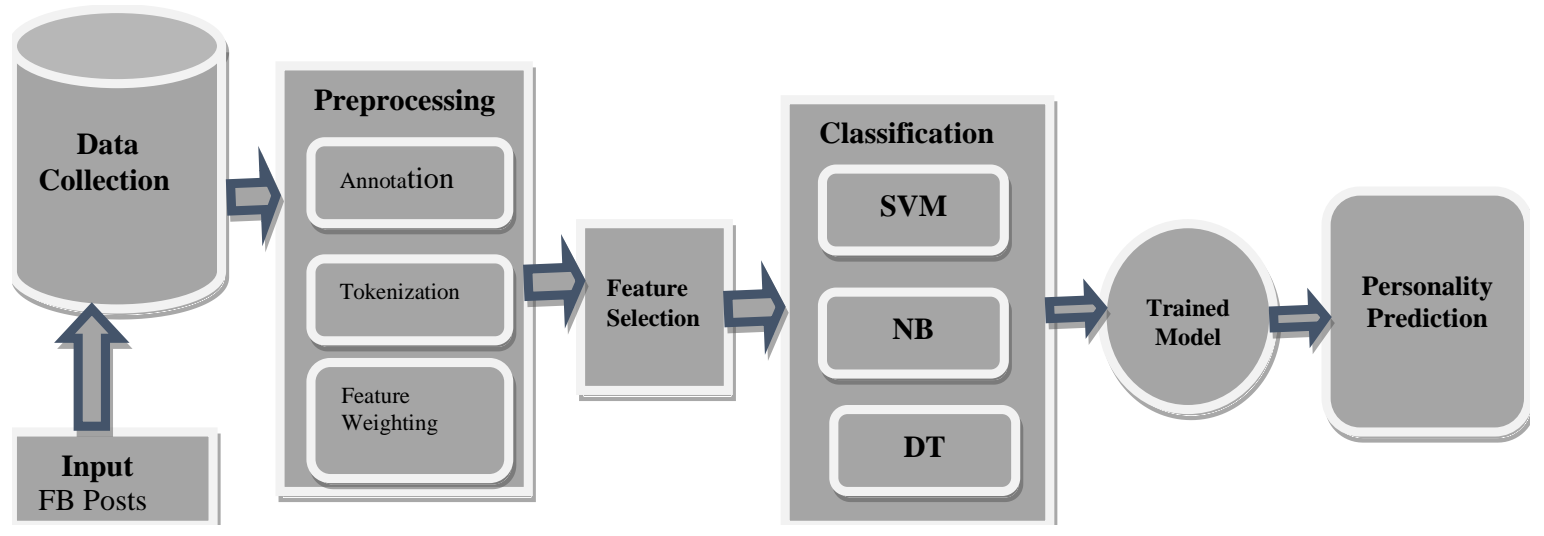

Fig.1. Personality Prediction Classification Process

Our work is based on quantitative and applied approaches. For this purpose, datasets have collected from Facebook using the Facebook Graph API [9].Personality traits have classified into five dimensions. These dimensions are extraversion, neuroticism, agreeableness, conscientiousness and open-ness. Our research approach is based on applied approach. It consists of collection of data, data representation, personality classification, personality traits prediction and results. In data collection process, data has been collected from Facebook using Graph API. Machine learning classifiers like naïve bays, support vector machine and Decision Tree are used for personality classification [27]. Further text mining techniques i.e. data preprocessing, tokenization and stemming are used for text classification. Personality traits have predicted using Personality Predication App that predicts personality traits after analyzing personality results. Moreover, results and comparison of these classifiers has evaluated. The classification process stages i.e. data collection, preprocess, feature selection, classification and personality prediction are briefly explained in section- 4 . 


\subsection{Behavioral Classification:}

The data is presented in order to determine the anti-social behavior used in tweets. Different machine learning classifiers has been used for the implementation of behavior predictor system. Supervised learning approach has been used in this research work. A brief introduction of Naïve Bays (NB) and Support Vector Machine (SVM) are efficient for our research problem [10]. These algorithms has been discussed in following section.

\section{Nä̈ve Bays Classifier}

In Naïve Bays, patterns are matched by examining set of categorized documents. It is a probabilistic classifier that matches the data with the bag of words. It streamlines the learning by classifying the features in an independent class. Naive Bay's accuracy is independent from the features dependencies. In [11] Text categorization can be viewed in the context of subsequent documents probabilities i.e. $P\left(c_{i} \mid d_{j}\right)$ where the probability of $\mathrm{j}^{\text {th }}$ document is represented in vectors. The weight vector is $d_{j}=<q 1_{j}, q 2_{j}, \ldots \ldots, q|T|_{j}>$ where $q_{k j}$ is the weight of kth feature in jth document is and belongs to class $c_{i}$. Naïve Bayes classifier is used to measure posterior probabilities which are as under:

$$
P\left(c_{i} \mid d_{j}\right)=\frac{P\left(d_{j} \mid c_{i}\right) P\left(c_{i}\right)}{P\left(d_{j}\right)}
$$

The posterior probability $c_{i}$ is used to select a random (arbitrary) document where $d_{j}$ is the probability of chosen arbitrary documents that has the weight vector $d \mathrm{j}$ and $P\left(d_{j} \mid c_{i}\right)$ this weight vector represents the probability of the document $d_{j}$ which is the member of class $c_{i}$. Though, the idea of $c_{i} \mid d_{j}$ is complex because the estimation of $d_{j} \mid c_{i}$ requires high dimensions of vector $d_{j}$. To sort this complex problem of calculations of $\left(d_{j} \mid c_{i}\right)$, it is supposed to have independent coordinates from document vector. This assumption leads to the term which is projected as:

$$
P\left(d_{j} \mid c_{i}\right)=\prod_{k=1}^{T} P\left(W_{k j} \mid c_{i}\right)
$$

\section{Support Vector Machine}

SVM works as a structural risk minimization. The major aim of this risk minimization is to determine a hypothesis to confirm minimum possible errors. According to the principles of risk minimization, training error and difficulty of hypothesis can be used for bounded true error. SVM is used to make resultant hypothesis free from true errors by maintaining its dimensions effectively. These dimensions can be represented in the context of Vapnik-Chervonekic or VC dimensions (Mitchell, 1997). Following is the mathematical solution that how to find the maximum margins:

$$
\text { minimize }_{w, b}=<w . w>
$$




$$
\text { Subject } y_{i}\left(<w \cdot x_{i}>+b\right) \geq 1 \quad \mathrm{i}=1 \ldots 1
$$

Here $x i$ is used as input vector and ' 1 ' exhibits training examples. Also $y_{i}$ is the required output. For ease, the above mathematical problem can be reformulated as:

$$
L(w, b, \alpha)=\frac{1}{2}<w \cdot w>-\sum_{i=1}^{1} \alpha_{i}\left[y_{i}\left(<w_{i} \cdot x_{i}>+b\right)-1\right]
$$

Where $\alpha_{i} \geq 0$ and $\alpha_{i}$ are langrage multipliers. This equation can be expressed in context of $\mathrm{w}$ and $\mathrm{b}$. After substituting values, new equation can be formulated as:

$$
L(w, b, \alpha)=\sum_{i=1}^{1} \alpha_{i}-\frac{1}{2} \sum_{i, j=1}^{1} y_{i} y_{j} \alpha_{i} \alpha_{j}<x_{i} x_{j}>
$$

The instance $x_{i}$ represents the new feature space of $x_{i}$. The dimensional space $x_{i}$ and $x_{j}$ are used for dual formulation of product which is the kernel functions.

\section{Data Collection and Experiments}

\subsection{Response Volume over Time}

To examine the traits of personality using Facebook data, we assessed 213 unique profiles. The average traits found in each category has been illustrated in fig. 2 .

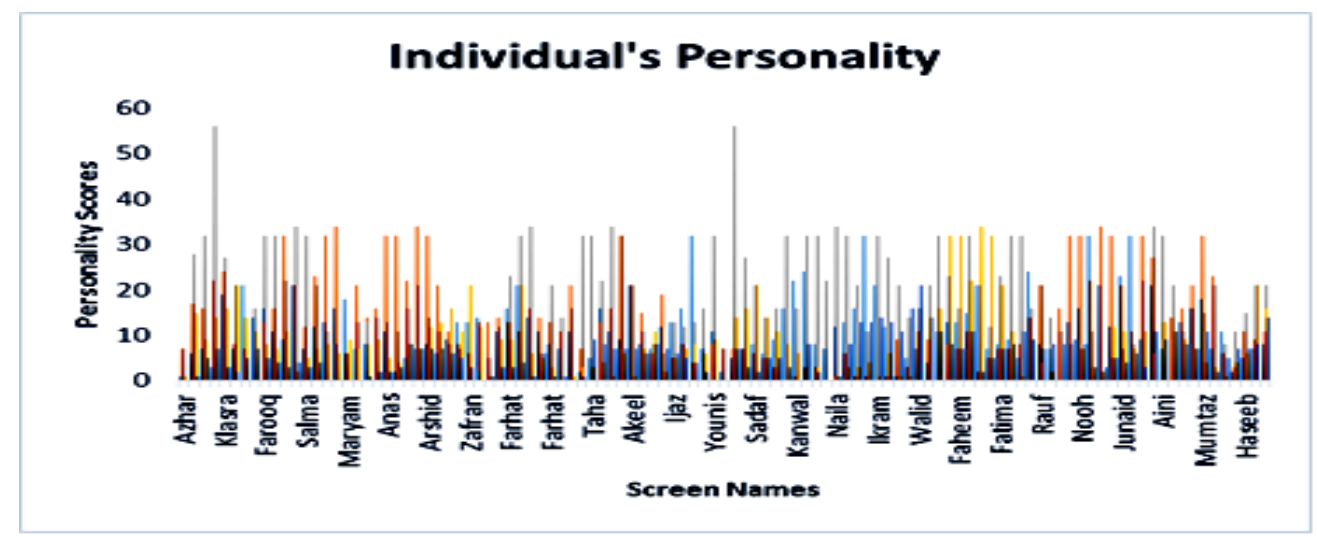

Fig.2. Personality Responses

Personality has analyzed with respect to five factors of personality including extraversion, neuroticism, agreeableness, openness and conscientiousness. The frequency of personality traits has described in fig.3. 


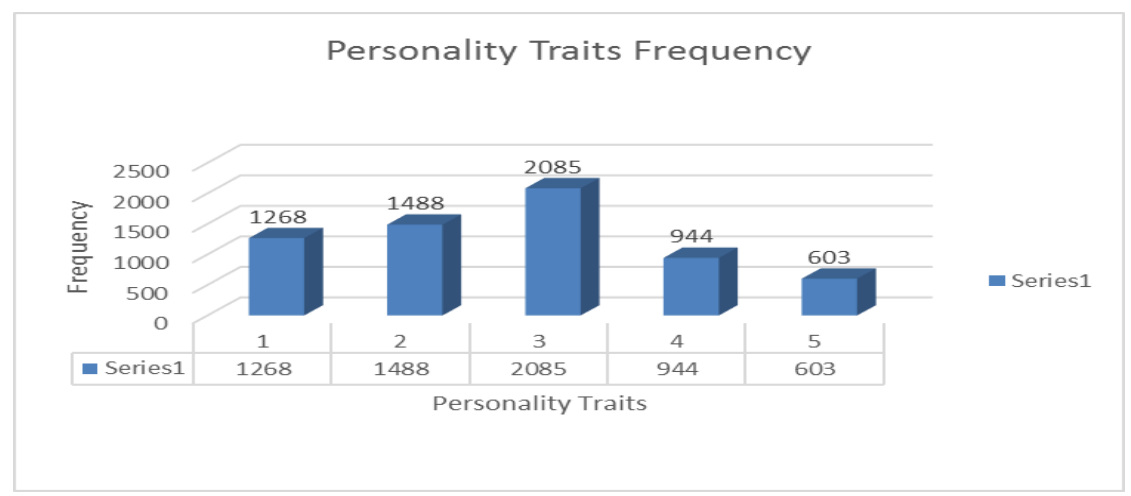

Fig.3. Frequency of Traits in Personality

\subsection{Personality Prediction Application}

There are different existing applications for analyzing personality. We have used in-house developed tool namely "Personality Predictor". This prediction app helps to predicts big 5 traits of personality using dictionary based approach. The interface of the in-house developed tool is given in fig.4.

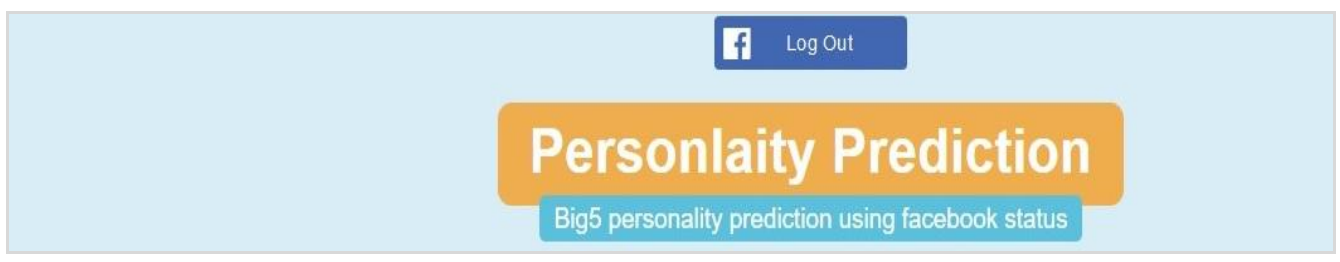

Fig.4. Interface of Personality Prediction Application

\subsection{Facebook Dataset}

Data collection is the primary step of our approach. For this purpose, 213 individual's Facebook profile data has been collected. The Facebook profile data has specified by using preprocessing, information extraction, tokenization, stop words removal and stemming. Personality of an individual has predicted by using one of the most popular dictionary SenticNet [12] [13]. To analyze personality traits, data from 213 unique profiles has been collected and assessed using Personality Prediction App as described in fig.5.

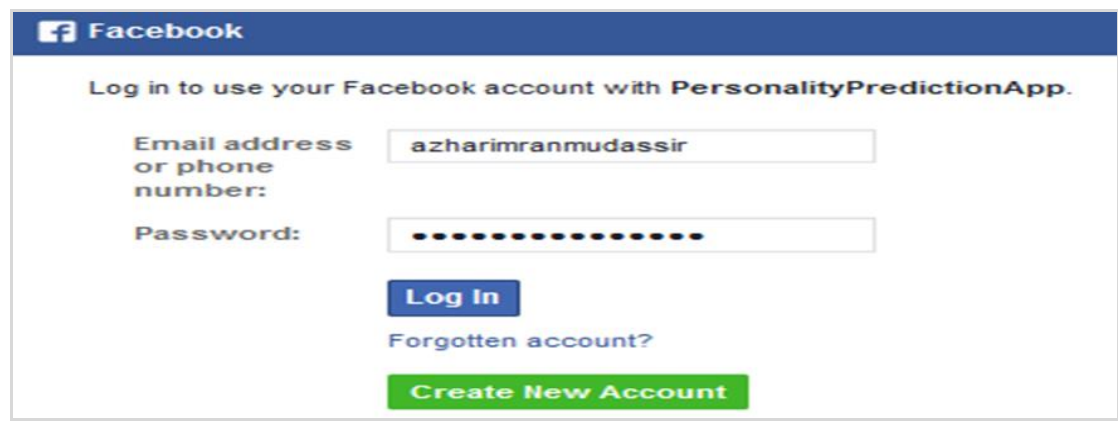

Fig.5. User's Login Permission 
After the confirmation from user, this application access user's profiles and public posts and then displays the big5 personality traits using dictionary based approach configured in it given in Fig.6.

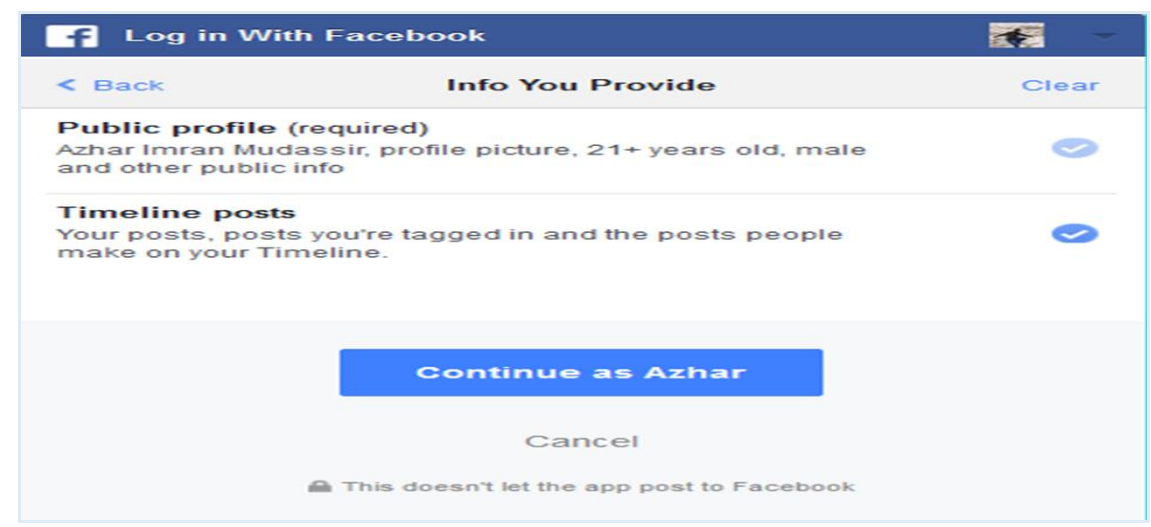

Fig.6. Data Usage Authentication

\subsection{Pre-Processing and Exemplification of Data}

In information portrayal, raw posts are changed into a configuration helpful for applying machine learning calculations. This includes feature representation (FR) and weighting. Tokenization is the process used for FR and TF-IDF is used to weight individual element [17]. The fundamental goal of preprocessing is information cleaning, separating and standardization. Information portrayal and pre-handling errands have vital significance in order to classify of textual information since legitimate portrayal and pre-preprocess enhance the viability of classifier as well as influence classifier to time productive.

\subsection{Feature Selection}

Feature selection is used for automatic selection of attributes pertinent to predictive modelling dataset. This decreases training time, improves accuracy and reduce over-fitting problems. Feature selection assigns a score to all features which is based on evaluation function [14]. Information Gain (IG) is the technique used to assign scores based on their features. IG uses term $t$ which is computed in given calculation.

$$
\left.\left.\left.I G(t)=\sum_{i=1}^{m} P c_{i}\right) \cdot \log P\left(c_{i}\right)+P(t) \sum_{i=1}^{m} P c_{i} \mid t\right) \cdot \log P\left(c_{i}\right)+P(t) \sum_{i=1}^{m} P c_{i} \mid t\right) \cdot \log P\left(c_{i} \mid t\right)
$$

Where $\mathrm{P}\left(\mathrm{c}_{\mathrm{i}}\right)$ denotes prior probability of categories set i.e. $\left(\mathrm{c}_{1}, \mathrm{c}_{2}, \mathrm{c}_{3} \ldots \ldots . \mathrm{c}_{\mathrm{n}}\right)$ and $\mathrm{P}(\mathrm{t})$ represents the prior probability of term.

\section{Analysis and Results}

The imperial results for the above dataset has been described in this section. After the successful login to the system, Personality Prediction application displays the results into five dimensions of personality which are Extraversion, Neuroticism, Agreeableness, Conscientiousness and Openness respectively. In fig.7. The data acquired from Facebook profiles has stored in Jason format with extension .JS. The following figure depicts that how the data has initially acquired for the later steps including data preprocessing and personality assessment. 


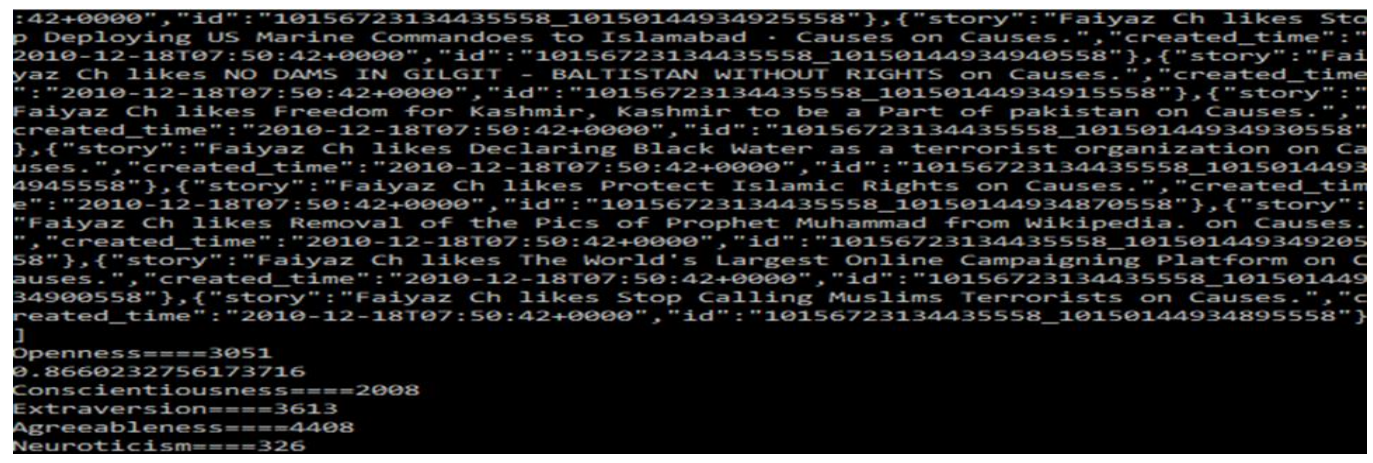

Fig.7. Personality Traits Results in JS format

Furthermore, we trained and test our data on WEKA tool (one of the largely tool available for research perspectives). We also used three classifiers i.e. SVM, naïve Bays and decision tree and effectiveness of each classifier is measured using precision, recall and F-measure. To validate results, we have used k-folded cross validation where training dataset is divided into k mutually exclusive subsets; interactively one of the $\mathrm{k}$ subset is used for testing the classifier and other k-1 subset used for training the classifier. We have conducted 10folded cross validation test to validate the accuracy of classifier. The percentage prediction values attained from conducting test is shown in fig.8.

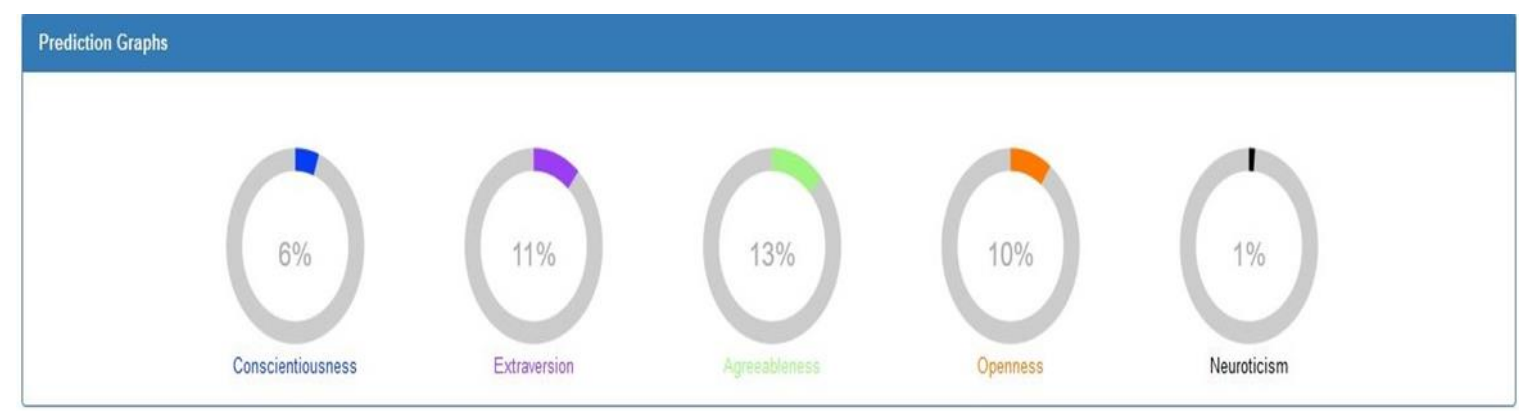

Fig.8. Personality Traits Result in Graph

Support Vector Machine (SVM), Naïve Bays (NB) and Decision tree (DT) are used for the classification of data (preprocessed). The accuracy of these classifiers is evaluated using model evaluation approaches precision, recall and f-measure. We have used 10 folded cross validation in Weka tool on testing and training dataset. Cross validation is a technique for model validation and it evaluates the generalization of independent data set over statistical results provided by model. We used precision, recall and f-measure to evaluate the performance of SVM, NB and LR as these standard evaluation measures have the ability to evaluate a category wise prediction of the classifiers. For the personality traits classes' precision, recall and f-measure can be defined as given in equation 6,7 and 8 respectively.

$$
\text { Precision }=\frac{\# \text { of Correct Positive Prediction }}{\# \text { of Positive Predictions }}
$$




$$
\begin{aligned}
& \text { Recall }=\frac{\# \text { of Correct Positive Prediction }}{\# \text { of Positive Examples }} \\
& F-\text { measure }=\frac{2 * \text { Precision } * \text { Recall }}{\text { Precision }+ \text { Recall }}
\end{aligned}
$$

The results of these classifiers are categorized into five personality trait classes i.e. Extraversion (EXT), Neuroticism (NEU), Agreeableness (AGR), Conscientiousness (CON) and Openness (OPE). The results obtained using SVM, NB and DT are illustrated in table 1, 2 and 3 respectively.

Table 1. Support Vector Machine Results

\begin{tabular}{|l|l|l|l|}
\hline Class & Precision & Recall & F-measure \\
\hline EXT & 0.743 & 0.999 & 0.852 \\
\hline NEU & 0.655 & 0.79 & 0.99 \\
\hline AGR & 0.968 & 0.969 & 0.987 \\
\hline CON & 0.927 & 0.779 & 0.847 \\
\hline OPE & 0.887 & 0.852 & 0.779 \\
\hline
\end{tabular}

Table 2. Naive Bays Results

\begin{tabular}{|l|l|l|l|}
\hline Class & Precision & Recall & F-measure \\
\hline EXT & 0.743 & 0.988 & 0.848 \\
\hline NEU & 0.885 & 0.674 & 0.759 \\
\hline AGR & 0.897 & 0.979 & 0.947 \\
\hline CON & 0.795 & 0.754 & 0.789 \\
\hline OPE & 0.895 & 0.654 & 0.789 \\
\hline
\end{tabular}

Table 3. Decision Tree Results

\begin{tabular}{|l|l|l|l|}
\hline Class & Precision & Recall & F-measure \\
\hline EXT & 0.743 & 0.999 & 0.852 \\
\hline NEU & 0.795 & 0.655 & 0.772 \\
\hline AGR & 0.985 & 0.874 & 0.959 \\
\hline CON & 0.868 & 0.869 & 0.887 \\
\hline OPE & 0.795 & 0.855 & 0.897 \\
\hline
\end{tabular}

The comparative analysis of three classifiers i.e. Support Vector Machine, Naïve Bays and Decision Tree has been performed. Model evaluation features precession, recall and F-measure has been used to find the accuracy of each classifier. The results of each classifiers appeared in Table.4. Although, results are same but it's shown the effectiveness of personality traits. 
Table 4. Comparative Analysis of Classifiers

\begin{tabular}{|l|l|l|l|}
\hline Classifiers & Precision & Recall & F-measure \\
\hline SVM & 0.987 & 0.982 & 0.982 \\
\hline NB & 0.986 & 0.982 & 0.981 \\
\hline DT & 0.987 & 0.982 & 0.982 \\
\hline
\end{tabular}

\section{Conclusions}

Personality traits replicate people's distinctive patterns of opinions, moods, and behaviors. Personality dimensions indicate regularity and solidity. In our research work we have predicted Big5 Personality traits using Facebook profile public timeline posts. Although, some work has been previously done on personality prediction of Facebook profile data in online discussion communities but to predict Big5 personality traits in Facebook was less targeted area. The concerns regarding prediction of Big5 Personality traits have implemented in our research work. Our work has shown decent results. We have presented a tool for Big5 Personality traits prediction. Personality prediction tool takes public profile data and timeline posts of a Facebook profile of an individual, then perform preprocessing, tokenization and stemming. Dictionary based approach has been used to analyze Big5 personality traits. Each trait results are shown in prediction Graph form.

Another facet of our research work is that we have implemented personality prediction feature using Facebook Graph API. It uses most informative features along with their personality traits. The comparative analysis of different machine learning classifiers such as SVM, Naïve Bays and Decision Tree has been done in our research work. SVM has shown the best results in personality prediction analysis.

\section{References}

[1] Van der Geer J, Hanraads JAJ, Lupton RA. The art of writing a scientific article. J Sci Commun 2000; 163:51-9.

[2] Obar, J. A., \& Wildman, S. S. (2015). Social media definition and the governance challenge: An introduction to the special issue.

[3] Kietzmann, J. H., Hermkens, K., McCarthy, I. P., \& Silvestre, B. S. (2011). Social media? Get serious! Understanding the functional building blocks of social media. Business horizons, 54(3), 241-251.

[4] Kuei-Hsiang, P., \& Li-Heng, L. (2015). Predicting personality traits of Chinese users based on Facebook wall posts. Wireless and Optical Communication Conference (WOCC), 2015.

[5] Krämer, N. C., \& Winter, S. (2008). Impression management 2.0.The relationship of self-esteem, extraversion, self-efficacy, and self-presentation within social networking sites. Journal of Media Psychology, 20(3): (pp. 106-112).

[6] Quercia, D., Lambiotte, R., Stillwell, D., Kosinski, M., \& Crowcroft, J. (2012). The personality of popular Facebook users. In Proceedings of the ACM 2012 conference on Computer Supported 32Cooperative Work, (pp. 955-964). ACM.

[7] Farnadi, G., Sitaraman, G., Sushmita, S., Celli, F., Kosinski, M., \& Stillwell, D. (2016, June). Computational personality recognition in social media, user modeling and user-adapted interaction. June 2016, Volume 26, Issue 2, (pp. 109-142).

[8] Farnadi, G., Zoghbi, S., Moens, M., \& De Cock, M. (2013). Recognising personality traits using Facebook status updates. In: Proceedings of the WCPR, (pp. 14-18).

[9] Quercia, D., Kosinski, M., Stillwell, D., \& Crowcroft, J. (2011). Our Twitter profiles, our selves: predicting personality with Twitter. In: Privacy, Security, Risk and Trust (passat), 2011 IEEE Third International Conference on Social Computing (socialcom), (pp. 180-185). IEEE. 
[10] https://developers.facebook.com/docs/graph-api

[11] Hsu, C. W., Chang, C. C., \& Lin, C. J. (2003). A practical guide to support vector classification.

[12] Rish, I. (2001, August). An empirical study of the naive Bayes classifier. In IJCAI 2001 workshop on empirical methods in artificial intelligence (Vol. 3, No. 22, pp. 41-46). IBM.

[13] http://sentic.net/senticnet-3.0.zip

[14] Cambria, E. (2016). Affective computing and sentiment analysis. IEEE Intelligent Systems, 31(2), 102-107.

[15] Ikonomakis, M., Kotsiantis, S., \& Tampakas,V. (2005). Text classification using machine learning techniques. WSEAS Transaction on Computers, 8(4), 966-974.

[16] Golbeck, J., Robles, C., Edmondson, M., \& Turner, K. (2011, October). Predicting personality from twitter. In Privacy, Security, Risk and Trust (PASSAT) and 2011 IEEE Third Inernational Conference on Social Computing (SocialCom), 2011 IEEE Third International Conference on (pp. 149-156). IEEE.

[17] Chen, T. Y., Chen, T. Y., Tsai, M. C., Tsai, M. C., Chen, Y. M., \& Chen, Y. M. (2016). A user's personality prediction approach by mining network interaction behaviors on Facebook. Online Information Review, 40(7), 913-937.

[18] Salton, G., \& Buckley, C. (1988). Term-weighting approaches in automatic text retrieval. Information Processing and Management, 24(5), 513-523, 1988.

[19] Google Search Statistics. (2016, January 10). Retrieved from website: http://www.internetlivestats.com/google-search-statsitics/.

[20] Vinay K. Jain, Shishir Kumar, "Towards Prediction of Election Outcomes Using Social Media", International Journal of Intelligent Systems and Applications(IJISA), Vol.9, No.12, pp.20-28, 2017. DOI: 10.5815/ijisa.2017.12.03

[21] Asad Mehmood, Abdul S. Palli, M.N.A. Khan,"A Study of Sentiment and Trend Analysis Techniques for Social Media Content", IJMECS, vol.6, no.12, pp.47-54, 2014.DOI: 10.5815/ijmecs.2014.12.07

[22] Ibtesam Fares. Al-Mashaqbeh,"Facebook Applications to Promote Academic Engagement: Student's Attitudes towards the Use of Facebook as a Learning Tool", IJMECS, vol.7, no.11, pp.60-66, 2015.DOI: 10.5815/ijmecs.2015.11.07.

[23] Waters, Richard D., et al. "Engaging stakeholders through social networking: How nonprofit organizations are using Facebook." Public relations review 35.2 (2009): 102-106.

[24] Courtney, K. L. "The use of social media in healthcare: organizational, clinical, and patient perspectives." Enabling health and healthcare through ICT: available, tailored and closer 183 (2013): 244.

[25] Zhang, Jie, Yongjun Sung, and Wei-Na Lee. "To play or not to play: An exploratory content analysis of branded entertainment in Facebook." American Journal of Business25.1 (2010): 53-64.

[26] Elmore, Kimberly L., et al. "mPING: Crowd-sourcing weather reports for research." Bulletin of the American Meteorological Society 95.9 (2014): 1335-1342.

[27] Sanderson, Jimmy. "From loving the hero to despising the villain: Sports fans, Facebook, and social identity threats." Mass Communication and Society 16.4 (2013): 487-509.

[28] Imran, A., W. Aslam, and M.I. Ullah. "Quantitative Prediction of Offensiveness using Text Mining of Twitter Data." Sindh University Research Journal-SURJ (Science Series) 49.1 (2017). 


\section{Authors' Profiles}

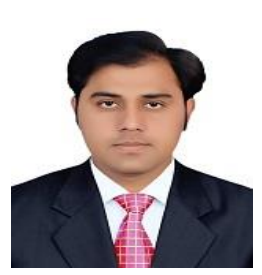

Azhar Imran is Doctoral Student in School of Software Engineering at Beijing University of Technology, China. He has received his Master degree in Computer Science in 2017 from University of Sargodha, Pakistan with distinction of Gold Medal. His research interests include Machine Learning, Data Mining and Deep Learnings.

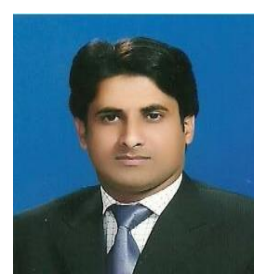

Muhammad Faiyaz is an Assistant Professor in Department of Computer Science at Govt. Municipal Degree College, Faisalabad, Pakistan. He has received his Master degree in Computer Science from University of Sargodha, Pakistan in 2017. His research interests include Data Mining, Information Retrieval, Knowledge Management, and Social Media Processing.

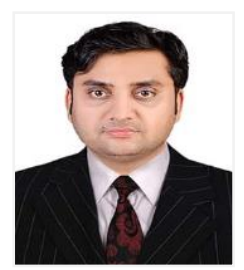

Faheem Akhtar is an Assistant Professor in Department of Computer Scienc at Govt. Institute of Business Administration, Sukkar, Pakistan. He has received his Master degree in Computer Science from NUCES FAST Karachi and BS in Computer Science from Hamdard University, Karachi. His research interests include Data Mining, Machine Learning, Knowledge and Neural Networks.

How to cite this paper: Azhar Imran, Muhammad Faiyaz, Faheem Akhtar,"An Enhanced Approach for Quantitative Prediction of Personality in Facebook Posts", International Journal of Education and Management Engineering(IJEME), Vol.8, No.2, pp.8-19, 2018.DOI: 10.5815/ijeme.2018.02.02 